\title{
Repoblamiento de macro algas en áreas de usos intensivos como oportunidad de recuperación de servicios ambientales en espacios degradados \\ MARCELO BAEZA SEQUEIRA
}

> Biólogo Marino Universidad de Valparaíso, Máster en Sistemas Integrados de Gestión Universidad Politécnica de Cataluña, Empresa Nacional de Petróleo - ENAP Aconcagua, Chile

mabaeza@enaprefinerias.cl

ORCID 0000-0003-2802-7849

Universidad de Valparaíso

Facultad de Arquitectura

Revista Márgenes

Espacio Arte Sociedad

Repoblamiento de macro algas en áreas de usos intensivos como oportunidad de recuperación de servicios ambientales en espacios degradados

Octubre 2021 Vol. $14 \mathrm{~N}^{\circ} 20$

Páginas 52 a 56

ISSN elec. 0719-4463

Recepción: Abril 2021

Aceptación: Abril 2021

doi.org/10.22370/marge-

nes.2021.14.20.2999
RESUMEN

Grandes esfuerzos se han realizado por parte del Estado para la protección de ecosistemas que prestan valiosos servicios ambientales, a través de diversas figuras de áreas protegidas, existiendo para ello marcos regulatorios en constante revisión. Estos se concentran en áreas que se puedan reservar para un uso preferente de conservación. Por otra parte, existen también intentos para la recuperación de especies que constituyen recursos económicos de los cuales dependen una comunidad o una actividad económica determinada. Pero cuando se trata de ecosistemas en lugares que soportan usos intensivos distintos a los servicios eco sistémicos originales no se ve una orgánica similar que vele por su cuidado o restauración. Un proyecto de repoblamiento de macroalgas en áreas de usos intensivo se presenta como una oportunidad de recuperación de servicios ambientales en espacios degradados, pero para su éxito debe sortear la dificultad de no contar con un marco regulatorio que avale dicha recuperación. Un cambio normativo que facilite e incentive la recuperación de ecosistemas es una oportunidad para resolver diversos conflictos ambientales.

PALABRAS CLAVE

ecosistema marino, macro algas, recuperación, recurso, políticas medioambientales, conservación, actividad económica

\section{Repopulation of macroalgae in areas of intensive use as an op- portunity to recover environmental services in degraded spaces}

\section{ABSTRACT}

Great efforts have been made to protect ecosystems that provide valuable environmental services, and its regulatory frameworks are being constantly reviewed and improved. These efforts are concentrated on areas that can be restricted for conservation purposes. There are also efforts to recover species that constitute economic resources which a community or a specific economic activity depends on. But when it comes to ecosystems in places that support intensive uses other than the original ecosystem services, there is not a similar framework that ensures their care or restoration. A macroalgae repopulation project in areas of intensive use is presented as an opportunity to recover environmental services in degraded environmental areas, but for its success it must overcome the difficulty of not having a regulatory framework that supports that recovery. $A$ regulatory change that facilitates and encourages the recovery of ecosystems is an opportunity to resolve various environmental conflicts.

\section{KEYWORDS}

marine ecosystem, macro algae, recovery, resource, environmental policies, conservation, economic activity 


\section{LOS ESFUERZOS POR CONSERVAR LOS SERVICIOS AMBIENTALES}

En los últimos años se han vistos grandes logros en la protección de espacios marinos y costeros en favor de la conservación de la biodiversidad de especies y se ha avanzado en el consenso de metas internacionales, cooperación y definición de modelos de gestión (Witte-Lebhar, 2017), procesos que en el caso de ecosistemas terrestres se han desarrollado con varias décadas de antelación, con éxitos y fracasos (Guerra Schleef, 2015; Mayorquín et al., 2010; Moreira-Muñoz \& Troncoso, 2014; Testa, 2013). Pero en ambos casos, el esquema es básicamente reservar un espacio físico para privilegiar la conservación y desarrollo de servicios ambientales (beneficios que obtienen las personas de los ecosistemas; Millenium Assessment, 2005) que se ven amenazados de diversas maneras, desde sobre explotación de recursos hasta cambio climático como también por el uso del territorio para el desarrollo de la vida humana (ciudades, carreteras, cultivos, industrias, etc.) (Millenium Assessment, 2005).

Cuando el espacio natural ya ha sido destinado para un uso antropocéntrico, por ejemplo: se ha transformado una bahía abrigada en un puerto, pareciera que se da por sentado que ya no presta más servicios ambientales y ahora es un espacio urbanizado que cumple un rol dentro de una función económica. Los puertos de Chile se consolidaron cuando el concepto de "externalidad negativa” aún no se acuñaba y no fue hasta la implementación de la evaluación de impacto ambiental que recién se incorporaron al análisis los impactos que generan la construcción y operación de actividades con usos intensivos del territorio (y maritorio).

Pero la evaluación de impacto ambiental solo aborda proyectos nuevos y exige medidas de gestión ambiental en los casos que se demuestre una clara relación de causa - efecto (Conesa, 2009), y el territorio no se empezó a urbanizar a partir de 1997 -que es cuando se inicia el Sistema de Evaluación de Impacto Ambiental en Chile-, luego, tenemos una serie de espacios naturales sufriendo las consecuencias de usos intensivos para los cuales la institucionalidad ambiental no tiene una respuesta e incluso en muchos casos ni siquiera una evaluación.

Cuando los recursos son escasos es comprensible que se concentren los esfuerzos en proteger espacios naturales que aún prestan valiosos servicios ambientales, estableciéndose como bastiones de resistencia ante otros usos intensivos por parte de los seres humanos. Para ello, los Estados establecen reservas y santuarios.

Cuando existen comunidades cuya subsistencia o sistema económico se basa en la explotación de ciertos recursos naturales, es comprensible que los esfuerzos se centren en promover la recuperación de dichos recursos, para que no colapse el sistema económico de la comunidad arrastrando a su vez el estilo de vida y la cultura de ésta, como es el caso más notorio de los riesgos que corren las comunidades de pescadores artesanales o las comunidades indígenas.

En cambio, cuando en el espacio natural ya se ha consolidado un uso intensivo, pareciera que en el solo quedan los elementos abióticos dando soporte físico a las actividades humanas que alli se desarrollan, como si el espacio abrigado dentro de un puerto, por ejemplo, fuera literalmente un desierto y ya no quedara ninguna especie del ecosistema original. En otras ocasiones, el desconocimiento de los sistemas naturales lleva a los tomadores de decisión a considerar espacios sin urbanizar como paños en blanco en dónde se puede proyectar cualquier intervención, ya que: "no hay nada”, sin embargo, la naturaleza está ahí, desarrollándose, resiliente pero invisible para muchos ojos.

Esta percepción de que estos espacios naturales han dejado de ser ecosistemas se va consolidado en el tiempo hasta que alguna disrupción los haga visibles, como, por ejemplo, algún proceso agudo de contaminación ambiental o un evento natural considerado catastrófico para las actividades humanas, como por ejemplo, un tsunami.

Son esos momentos lo que permiten aunar esfuerzos para reparar y/o reconstruir lo dañado, gracias a lo cual se generan mandatos, se habilitan recursos y se selecciona capital humano para ello, pero que al momento de ponerse manos a la obra corren severos riesgos de toparse con una dificultad muy particular en el caso de los espacios naturales: las autoridades que tienen bajo su competencia o jurisdicción la administración de dichos espacios naturales, no poseen instrumentos para autorizar una intervención en el territorio cuyo objetivo sea la recuperación del ecosistema per se, si en este proceso no se contemplan recursos naturales que sustenten una actividad económica.

\section{REPOBLAMIENTO DE ALGAS PARDAS (KELPS) EN LA BAHÍA DE QUINTERO}

Diversos proyectos con algas se han realizado en la bahía de Quintero en el último tiempo (Aqua.cl, Ciencia.Unab.cl, PlataformaArquitectura.cl), principalmente de investigación y ensayo de innovaciones, en un contexto de conflictos ambientales con diversas aristas y el esfuerzo de la institucionalidad por mantener una mesa de trabajo en donde participan líderes sociales, las empresas, los representantes de los municipios y el Gobierno en ejercicio (CRAS: Comité de Recuperación Ambiental y Social, vigente desde el 2014). Diversos proyectos también se han realizado en las áreas de manejo del sector, a fin de mejorar su productividad, ante la duda legítima de los pescadores artesanales respecto a la viabilidad de comercializar dichos recursos.

Uno de los proyectos en marcha, financiado por la Empresa Nacional del Petróleo, ENAP, consiste en un programa de remediación para la bahía de Quintero mediante la repoblación de organismos bio-remediadores pertenecientes al primer nivel trófico, que busca aumentar la capacidad de resiliencia del ecosistema. Dicho proyecto emana de una transacción entre la empresa y la Municipalidad de Quintero ante el Segundo Tribunal Ambiental a consecuencia del accidente de la nave Ikaros que derramó slurry oil (un hidrocarburo pesado) al fondo de la Bahía de Quintero.

Los objetivos de este programa de recuperación ambiental apuntan a la rehabilitación del ecosistema, en un proceso distinto al repoblamiento en áreas de manejo con fines de fomento productivo. En este caso, no se busca cosechar las algas sino generar servicios ambientales de regulación y soporte para las demás poblaciones de especies de la bahía, lo cual en el mediano y largo plazo tendrá como consecuencia secundaria repercusiones positivas en la productividad de las áreas de manejo.

Dicho de otra manera, las macroalgas al crecer forman verdaderos bosques submarinos (kelps) que se transforman en el hábitat de peces, moluscos, crustáceos, equinodermos y otros grupos de especies marinas que encuentran refugio y alimentación entre las frondas y grampones de estas algas pardas (feófitas). 


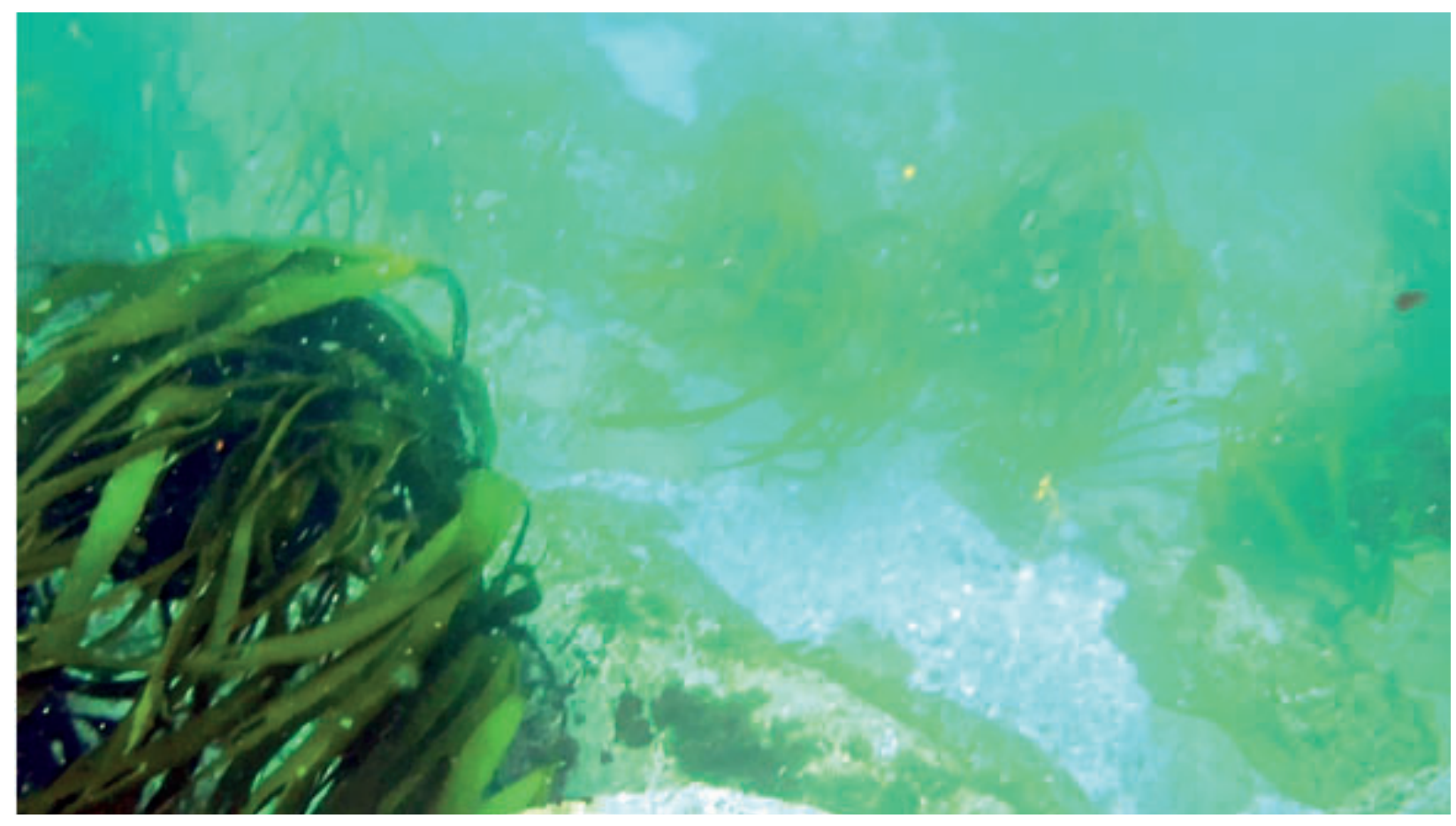

Chile cuenta con muchas experiencias exitosas de repoblamiento de algas (Ávila et al., 2012), no solo feófitas, ya que es un recurso importante para la pesca artesanal, por lo cual existe regulación (Ley N 18.892 Ley General de Pesca y Acuicultura) y recursos para incentivar iniciativas al respecto (Ley $\mathrm{N}^{\circ} 20.925$ Crea Bonificación para el Repoblamiento y Cultivo de Algas), pero siempre en un contexto productivo y de preferencia en un área de manejo de recursos bentónicos.

Pero cuando el objetivo es recuperar el ecosistema, que además no es área de manejo, dentro de una bahía de uso preferente portuario, como es en este caso en particular, la autoridad competente respecto a la administración de los recursos naturales marinos, la Subsecretaría de Pesca, no dispone de un permiso que autorice la intervención del territorio para estos fines, con lo cual los ejecutantes se ven forzados a buscar fórmulas dentro de la institucionalidad vigente, debiendo modificar los objetivos iniciales incorporando elementos que apunten a la producción y cosecha de una biomasa determinada de algas, que a veces es precisamente lo que se quiere evitar (cosechar) durante el desarrollo del proyecto.

Uno de los conflictos que presenta esta readecuación de los objetivos son los distintos horizontes de tiempo en los cuales se espera un resultado óptimo, mientras los procesos de siembra o repoblamiento en áreas de manejo esperan dar resultados en el corto plazo (una o dos temporadas dependiendo de la especie en cuestión), el repoblamiento con fines de remediación ambiental mostrará resultados en el mediano y largo plazo.

Por otra parte, los ciclos constantes de repoblar y cosechar pueden terminar en una rutina eterna que puede que le de sustento temporal a la actividad económica asociada al recurso pero no permite que el recurso como tal y su ecosistema se recuperen y se auto sustenten (Alfonso, 2020).

$>$ Figuras 1 y 2. Poblamientos de algas en zona de Quintero. Fuente: Registro fotográfico del autor. 


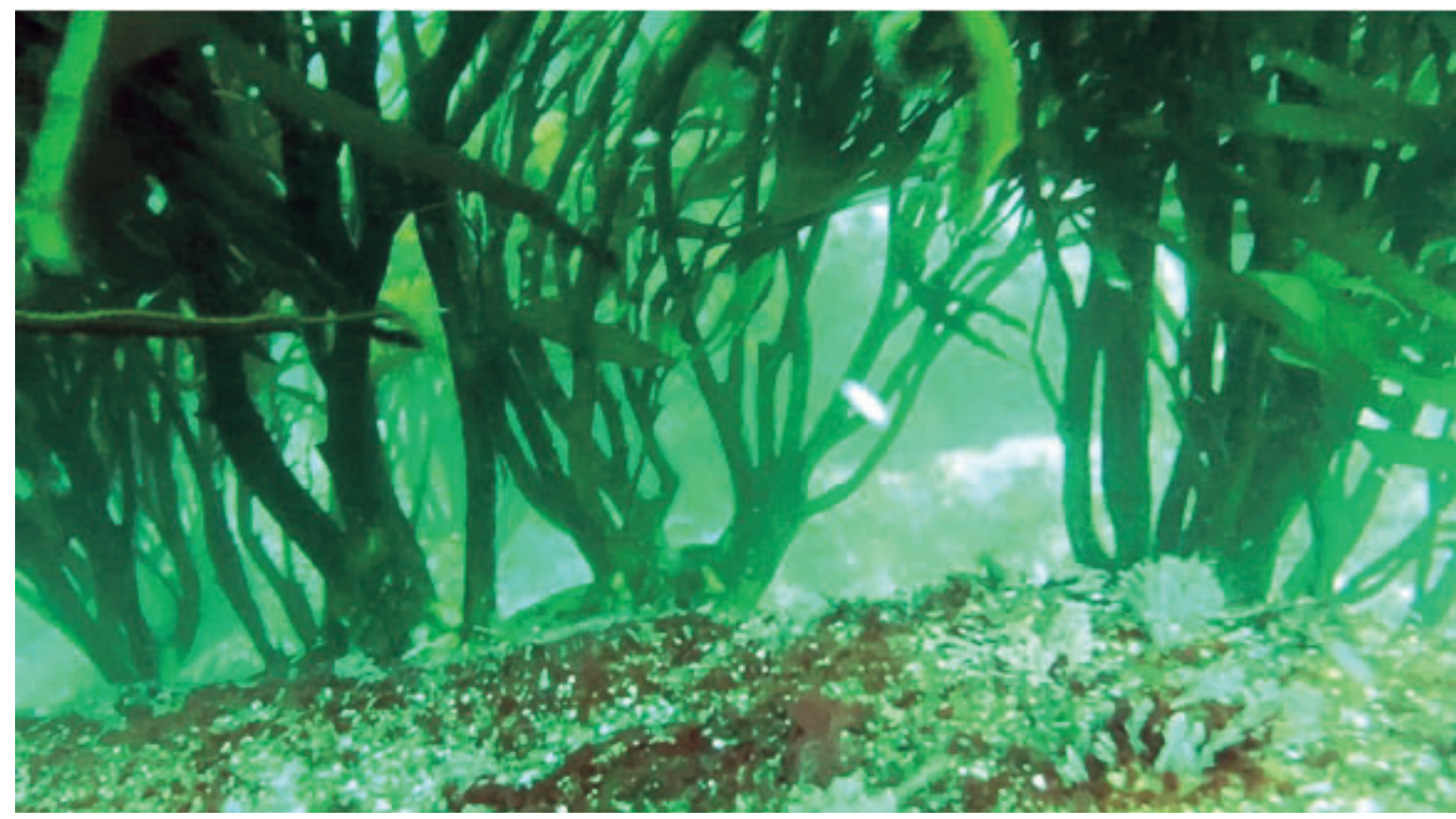

\section{LA OPORTUNIDAD QUE OFRECE LA RECUPERACIÓN DE ECOSISTEMAS}

Así como ENAP posee este mandato de desarrollar un proyecto de repoblamiento de macroalgas en un área de su operación logística, es probable que en lo sucesivo diversos conflictos ambientales se resuelvan con iniciativas similares, en donde se ponga en valor el recuperar los servicios ambientales de los ecosistemas que dan soporte a actividades económicas varias, en particular las industriales.

Intervenciones puntuales pero regulares en un territorio terminan por configurar un programa consistente de recuperación ambiental que puede permitirles a diversos ecosistemas superar un umbral de estrés que los conduzca claramente a un nuevo estado de madurez, que soporte de mejor manera perturbaciones tanto naturales como antrópicas (Simenstand et al., 2006). Se entiende que una red con mayor cantidad de nudos e hilos puede soportar mayor presión sin romperse.

Los ecosistemas ofrecen simultáneamente varios servicios ambientales, luego un proyecto de repoblamiento de algas no solo dará provisión y soporte a otras poblaciones de su comunidad biológica como ya se ha mencionado, sino también permitirá regular en el medio receptor la cantidad y efectos de las descargas industriales a los cuerpos de aguas sean sales, metales pesados o energía calórica, diversas investigaciones se han realizado al respecto (Aminina \& Shaposhnikova, 2012; Davis et al., 2003; Evans \& Edwards, 2011; Chung et al., 2013; Farías et al., 2018; Sáez et al., 2012, 2015).

La recuperación de estos servicios ambientales ofrece importantes sinergias para otros ecosistemas que tengan algún grado de protección y bajo ese concepto, es altamente probable que se beneficien de éstos la amplia red de áreas de manejo de recursos bentónicos que existe en el país.

Luego, la recuperación de ecosistemas en áreas de libre acceso (es decir que no están bajo el régimen de administración de un área de manejo) es también una herramienta indirecta para el fomento productivo de las áreas de manejo cercanas. A mayor trama trófica y menor volumen o concentración de estresores habrá mayor productividad.

\section{LA NECESIDAD DE UN CAMBIO REGLAMENTARIO PARA LA JUSTICIA AMBIENTAL}

Si bien cada conflicto ambiental debe tener una resolución en su propio mérito, también es dato de la causa que muchas veces es difícil establecer una relación clara de causa - efecto en estos, al ser los procesos ambientales multifactoriales y que además se desarrollan en diversas escalas temporales y espaciales.

La Autoridad Ambiental tiene la oportunidad de gestionar un marco regulatorio junto a los demás organismos sectoriales que facilite e incentive la recuperación de ecosistemas degradados como una alternativa válida de resolución de conflictos zanjados por la justicia ambiental, para que así las medidas validadas desde el Poder Judicial tengan efectivamente una posibilidad de resolución oportuna en el territorio afectado.

Resulta una paradoja que una institución del Estado mandate a una industria a una remediación ambiental y que otra institución del mismo Estado no posea una orgánica para autorizar dicha remediación, es decir, no se puedan obtener los necesarios permisos sectoriales (para una actividad que no cumple los requisitos para ingresar al Sistema de Evaluación de Impacto Ambiental) para intervenir en el territorio.

Para la correcta protección de bienes públicos, como lo es el mar, es necesaria una regulación, dado que en derecho público solo se puede hacer lo que está escrito y la existencia de un vacío normativo no posibilita monitorear y controlar los efectos de este tipo de remediaciones, ya que la autoridad no tiene claridad de cómo actuar frente a estas innovadoras soluciones.

Estas líneas son una invitación, a quien corresponda, para que dicha reglamentación exista.

\section{AGRADECIMIENTOS}

Quiero expresar mi gratitud a los colegas y amigos que desinteresadamente hicieron sus aportes y comentarios para mejorar la presente publicación: Marcela, Eduardo, Paola y Juan. 


\section{BIBLIOGRAFÍA}

Alfonso, Fernanda (2020) Evaluación de impacto para la acción de repoblamiento del recurso pesquero loco (Concholepas concholepas). Tesis (Magíster en Recursos Naturales) Pontificia Universidad Católica de Chile, 2020. Disponible en: https://repositorio.uc.cl/handle/11534/48219

Aminina, N.M. \& T.V. Shaposhnikova (2012) Peculiarities of growth and metabolism in japanese kelp in hábitats exposed to chronic contamination. Research in Plant Biology, 2012, 2(1):32-40.

Ávila M, C Godoy, D Rodríguez (2012) Manual para la repoblación de algas: desde la extracción hacia la agronomía marina. Resultados y lecciones. Serie Programa Educativo Participativo para la Pesca Artesanal. I La repoblación de lugas. Universidad Arturo Prat 70 pp.

Chung, Ik Kyo; Oak, Jung Hyun; Lee, Jin Ae Shin, Jong Ahm; Kim, Jong Gyu; Park, Kwang-Seok . 2013. Installing kelp forest/ seaweed beds for mitigation and adaptation against global warming: Korean Project Overview. ICES Journal of Marine Science 70(5), 1038-1044. https://doi.org/10.1093/icesjms/fss206 Disponible en: https://academic.oup.com/ icesjms/article/70/5/1038/644026

Conesa Fernández-Vitoria, Vicente (2009) Guía metodológica para la evaluación del impacto ambiental. Ediciones Mundi-Prensa. España

Davis, T. Volesky, B. \& A. Mucci (2003) A review of the biochemistry of heavy metal biosorption by brown algae. Water Research 37 (2003):4311-4330.

Evans, L.K. \& M.S. Edwards (2011) Bioaccumulation of cooper and zinc by the giant kelp Macrocystis pyrifera. Algae 2011, 26(3):265-275.

Farias, D.R., Hurd, C.L., Eriksen, R.S. \& C.K. Macleod (2018) Macrophytes as bioindicators of heavy metal pollution in estuarine and coastal environments. Marine Pollution Bulletin 128 (2018):175-184.

Guerra Schleef, Felipe (2015) El caso Tres Bocas y su importancia para la institucionalidad implicada en la gestión del Santuario de la Naturaleza del río Cruces (Corte Suprema). Revista de Derecho (Valdivia), 28(1), 253263. Disponible en: https://dx.doi.org/10.4067/S071809502015000100012

Mayorquín, Adriana; Valenzuela, Sandra y Rangel, Jesús Orlando. 2010. «Evaluación de la efectividad de manejo en reservas naturales de la sociedad civil: una propuesta metodológica». Caldasia 32 (2). Disponible en: https://revistas.unal. edu.co/index.php/cal/article/view/36230

Millenium Assessment (2005) Ecosystemas and Human Well. being. Synthesis. Island Press. Washington.

Moreira-Muñoz, A. \& J. Troncoso (2014) Representatividad biogeográfica de las Reservas de la Biosfera de Chile. En: A Moreira-Muñoz \& A Borsdorf (eds) Reservas de la Biosfera de Chile: Laboratorios para la Sustentabilidad. Academia de Ciencias Austriaca, Pontificia Universidad Católica de Chile, Instituto de Geografía, Santiago, serie Geolibros 17:24-61
Sáez, Claudio; Lobo M. Gabriela; Macaya Erasmo C.; Oliva, Doris; Quiroz Waldo, et al. 2012. Variation in patterns of metal accumulation in thallus parts of Lessonia trabeculata (Laminariales; Phaeophyceae): implications for biomonitoring. PLos ONE 7 (11): e50170. Diponible en: https:// journals.plos.org/plosone/article?id=10.1371/journal. pone.0050170

Sáez, C., Roncarati, F., Moenne, A., Moody, A.J. \& M. Brown (2015) Cooper-induced intra-specific oxidative damage and antioxidant responses in strains of the brown alga Ectocarpus siliculosus with different pollution histories. Aquatic Toxicology 159 (2015):81-89.

Simenstand, C., Reed, D. \& M. Ford (2006) When is restoration not? Incorporating landscape-scale processes to restore self-sustaining ecosystems in coastal wetland restoration. Ecological Engineering 26 (2006):27-39.

Testa, Joaquín (2013) Una aproximación para la evaluación técnica y operativa de las áreas naturales protegidas. El caso de las reservas costeras de la provincia de Buenos Aires, Argentina. RIAT: Revista Interamericana de Ambiente y Turismo. Vol. 9, $\mathrm{N}^{\circ}$ 2, pp. 86-100, 2013.

Witte-Lebhar, Benjamin (2017) Marine Reserves Announced for Rapa Nui and Other Chilean Islands. Disponible en: https://digitalrepository.unm.edu/notisur/14557

\section{LINKOGRAFÍA}

AQUA: Acuicultura más pesca: En: https://www.aqua. cl/2015/05/06/bahia-de-quintero-ya-puede-trabajar-enun-proyecto-de-biorremediacion-con-algas/

Centro para la Comunicación de la Ciencia. En: https://ciencia. unab.cl/investigadores-unab-demuestran-capacidad-de-algas-para-absorber-contaminantes-en-ambientes-marinos/

Revista de Arquitectura Digital: Plataforma Urbana. En: https:// www.plataformaarquitectura.cl/cl/904004/proyectouniversitario-busca-bioremediar-la-bahia-de-quintero-zonade-sacrificio-costera-en-chile 